\title{
Global prevalence of classic phenylketonuria based on Neonatal Screening Program Data: systematic review and meta-analysis
}

\author{
Hamid Reza Shoraka, $\mathrm{PhD}^{1}$, Ali Akbar Haghdoost, MD, $\mathrm{PhD}^{2}$, Mohammad Reza Baneshi, PhD ${ }^{3}$, Zohre Bagherinezhad, \\ $\mathrm{PhDc}^{4}$, Farzaneh Zolala, $\mathrm{PhD}^{5}$ \\ ${ }^{1}$ Health Services Management Research Center, Institute for Futures Studies in Health, Kerman University of Medical Sciences, Kerman, Iran; ${ }^{2} \mathrm{HIV} / \mathrm{STI}$ Surveillance \\ Research Center, and WHO Collaborating Center for HIV Surveillance, Institute for Futures Studies in Health, Kerman University of Medical Sciences, Kerman, Iran; \\ ${ }^{3}$ Modeling in health research center, Institute for futures studies in health, Kerman University of medical sciences, Kerman, Iran; ${ }^{4}$ Department of Medical Library and \\ Information Science, Kerman University of Medical Sciences, Kerman, Iran; ${ }^{5}$ Social Determinants of Health Research Center, Institute for Futures Studies in Health, \\ Kerman University of Medical Sciences, Kerman, Iran
}

Phenylketonuria is a disease caused by congenital defects in phenylalanine metabolism that leads to irreversible nerve cell damage. However, its detection in the early days of life can reduce its severity. Thus, many countries have started disease screening programs for neonates. The present study aimed to determine the worldwide prevalence of classic phenylketonuria using the data of neonatal screening studies. The PubMed, Web of Sciences, Sciences Direct, ProQuest, and Scopus databases were searched for related articles. Article quality was evaluated using the Joanna Briggs Institute Critical Appraisal Evaluation Checklist. A random effect was used to calculate the pooled prevalence, and a phenylketonuria prevalence per 100,000 neonates was reported. A total of 53 studies with $119,152,905$ participants conducted in 1964 2017 were included in this systematic review. The highest prevalence (38.13) was reported in Turkey, while the lowest $(0.3)$ in Thailand. A total of 46 studies were entered into the meta-analysis for pooled prevalence estimation. The overall worldwide prevalence of the disease is 6.002 per 100,000 neonates (95\% confidence interval, 5.07-6.93). The metaregression test showed high heterogeneity in the worldwide disease prevalence $\left(I^{2}=99 \%\right)$. Heterogeneity in the world wide prevalence of phenylketonuria is high, possibly due to differences in factors affecting the disease, such as consanguineous marriages and genetic reserves in different countries, study performance, diagnostic tests, cutoff points, and sample size.

Keywords: Phenylketonuria, Prevalence, Neonates, Screening, Meta-analysis

\begin{abstract}
Key message
Question: What is the global prevalence of classic phenylketonuria based on Neonatal Screening Program Data?

Finding: The overall worldwide prevalence of the disease is 6.002 per 100,000 neonates. The highest prevalence (38.13) was reported in Turkey, while the lowest $(0.3)$ in Thailand.

Meaning: This difference in the prevalence may be due to differences in the number of consanguineous marriages among the different regions, phenylalanine cutoff points, and sample sizes.
\end{abstract}

\section{Introduction}

Genetic and congenital abnormalities are the most important causes of death and malformation in the first month of life. ${ }^{1)}$ Phenylketonuria (PKU) is an inborn error of amino acid metabolism caused by phenylalanine hydroxylase gene mutations. ${ }^{2,3)}$ PKU patients experience an irreversible decrease in intelligence quotient scores, suppressed verbal function, impaired attention, and underdeveloped motor control skills. ${ }^{4,5)}$

The early diagnosis of PKU before the end of the first month of life is critical to controlling hyperphenylalaninemia. ${ }^{4,6)}$ Children with PKU seem normal during the first days of life; however, nervous system damage progresses gradually and becomes apparent over several months. ${ }^{7)}$ The early detection of PKU in the asymptomatic period and treatment with a phenylalanine restricted diet is warranted to ensure normal development. ${ }^{8-12)}$ Therefore, neonatal screening as a fundamental public health intervention started in the mid-20th century. ${ }^{4,12,13)}$

Since PKU has autosomal recessive inheritance, consanguineous marriage is an important risk factor ${ }^{1,4)}$; thus, countries with a high prevalence of consanguineous marriages have high

\footnotetext{
Corresponding author: Farzaneh Zolala, PhD. Institute for Futures Studies in Health, Kerman University of Medical Sciences, Medical University Campus, Haft-Bagh Highway, Kerman, Iran 


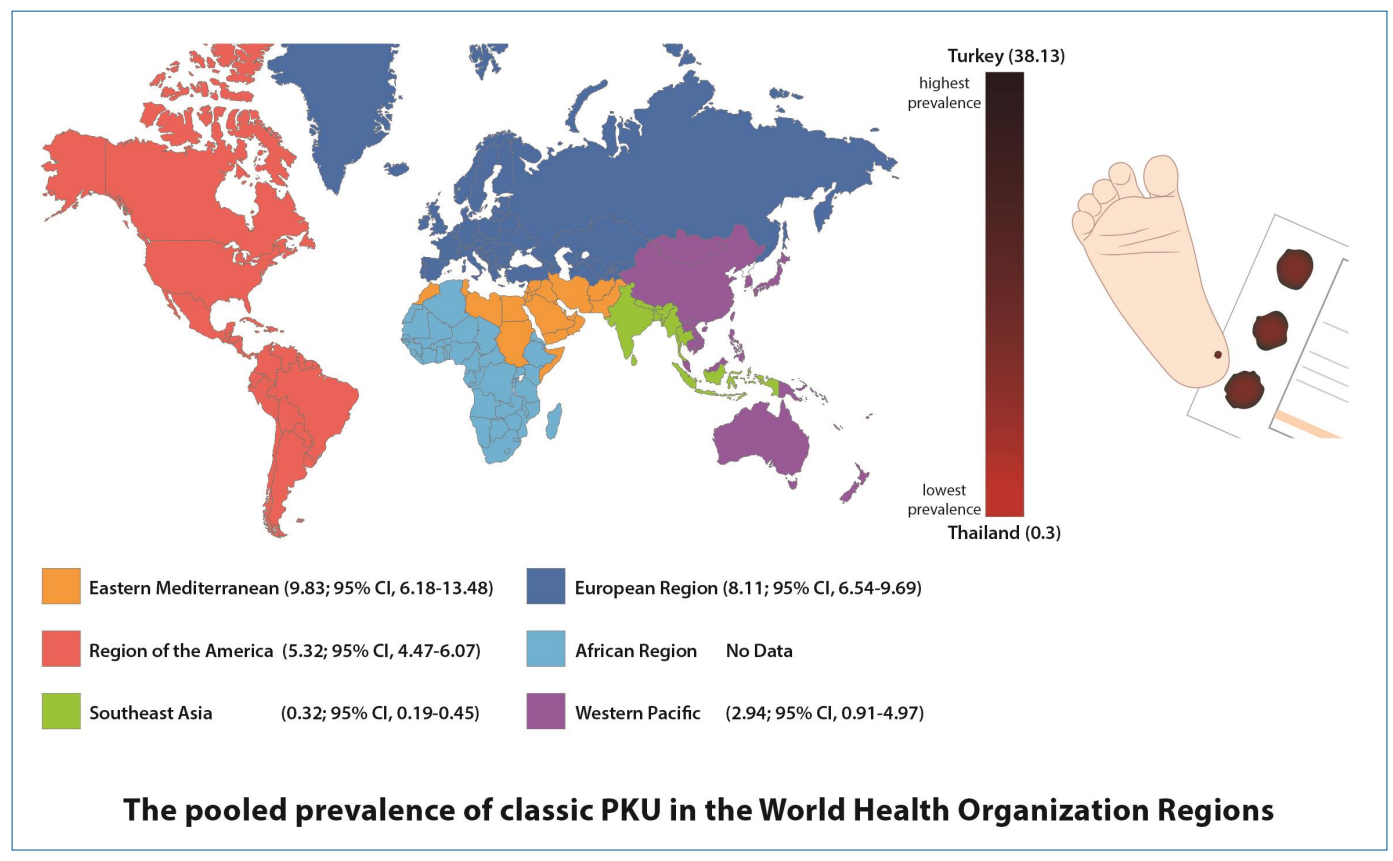

disease prevalence. ${ }^{6,14)}$ PKU varies among ethnic groups, races, and geographic regions. For example, In Japan, the incidence is reportedly $1: 108,822 .{ }^{15)}$ Turkey, with an incidence of $1: 6,000$, and Iran, with an incidence of 1:4,698, are among the countries with the highest PKU incidences. ${ }^{16,17)}$

Despite numerous studies conducted in various countries on PKU prevalence using screening programs, no study has systematically compared the prevalence of PKU across regions and countries or sources of heterogeneity. To address this gap, this systematic review and meta-analysis aimed to investigate the worldwide prevalence of PKU. Moreover, many countries have acknowledged the benefits of newborn screening programs for PKU. Moreover, newborn screening programs have enabled the rapid and large-scale testing of many children with good quality control.

\section{Methods}

This systematic review adhered to the guidelines of the Joanna Briggs Institute Reviewers' Manual 2014, Systematic Review of Prevalence Data. ${ }^{18)}$ The PRISMA (Preferred Reporting Items for Systematic Reviews and Meta-Analyses) 2009 flow diagram was used to guide the study identification and selection process. ${ }^{19)}$

\section{Search method}

The PubMed, Web of Sciences, Sciences Direct, ProQuest, and Scopus databases were searched on Oct 28, 2018, without publication date restrictions. The search strategy carefully captured all potentially eligible records of PKU prevalence. A combination of medical subject headings $(\mathrm{MeSH})$ and similar text words in English was used. Google Scholar was searched, as were the reference lists of the reviewed articles to identify additional relevant articles.

The key MeSH terms were as follows: (Infants OR Newborns OR Neonate) AND (Phenylketonuria OR Hyperphenylalaninemia, Non-Phenylketonuric OR BH4 Deficiency OR Tetrahydrobiopterin Deficiency OR Phenylketonuria II OR DHPR Deficiency OR Dihydropteridine Reductase Deficiency OR Atypical PKU) AND (Incidence OR Prevalence) and Screening. No time limitation was considered for the database search.

\section{Inclusion criteria}

All original articles that directly reported PKU prevalence based on newborn screening of populations were included. A newborn, infant, or neonate is a child younger than 28 days of age; in this review, the sampling period was limited to the first 28 days of life. All studies were included if they used a laboratory screening test for disease detection.

Reviews, comments, and letters were excluded. Moreover, studies that reported the prevalence in a selective neonatal population (congenital diseases, intellectual disability), those that included children older than 28 days, and those that indirectly estimated prevalence according to consanguinity or the incidence of another genetic disease were excluded. In some cases, studies were conducted of the same PKU prevalence in one country using different dates; in such cases, the more recent study (which also included the data from the older study) was included and the older study was omitted. Moreover, studies that detected PKU based on clinical manifestations in neonates or neural tube defects in fetuses were excluded.

\section{Data collection}

The title, abstract, and keywords of every identified article 
were carefully scanned and relevant articles were selected by title or abstract review.

\section{Data extraction and management}

Two reviewers ( $\mathrm{HSH}$. and FZ) independently extracted the patient characteristics, study characteristics, screening test used, and incidence from the reviewed studies using a data extraction form. Any disagreements between the 2 researchers were solved by consultation of another reviewer or a clinical adviser. Abstracts not published as full texts were not included in the study. To avoid data entry errors, all results were double-entered into a data extraction form. The included studies used different units such as $\mathrm{mmol} / \mathrm{L}, \mu \mathrm{mol} / \mathrm{dL}$, and $\mathrm{mg} / \mathrm{dL}$ to report phenylalanine level. However, in this study, to increase comparability, all units were converted to $\mathrm{mg} / \mathrm{dL}$. In addition, to ensure more accurate comparisons, PKU prevalence was calculated as percentage and rate per 100,000 screened neonates.

\section{Assessment of methodological quality}

Article quality was assessed using the Joanna Briggs Institute Critical Appraisal Checklist for studies that reported prevalence data. Each article was evaluated according to the following methodological criteria: appropriate sample, adequate sample size, valid methodology, valid measure to detect the disease, and an appropriate statistical analysis.

\section{Risk of bias}

Risk of bias was assessed using the risk of bias tool for studies measuring disease prevalence designed and developed by Hoy et al. ${ }^{20)}$ Based on this 10-point checklist, studies were assessed for internal and external validity and grouped as having high, moderate, or low bias risk. Studies with a score of 9-10 were considered at having low risk of bias; $6-8$, as having moderate risk; and less than 6 , as having high risk. Those studies with a high risk of bias were excluded from the meta-analysis.

\section{Data analysis}

The data were analyzed using Stata ver. 12 (StataCorp LP., College Station, TX, USA). In a meta-analysis, pooled prevalence was estimated based on World Health Organization (WHO) regions and reported as per 100,000 neonates/population with 95\% confidence interval (CI).

The degrees of heterogeneity among the included studies are expressed by the $I^{2}$ heterogeneity statistic, and the random effects model was used to estimate the pooled prevalence in subgroups. A forest plot was used to display the meta-analysis results.

The mixed model test considered WHO regions as a random intercept. In this test, phenylalanine levels were modeled as independent variables, while prevalence was considered a dependent variable.

Furthermore, a meta-regression analysis was performed to investigate the impact of variables such as WHO region, phenylalanine cutoff point, study period, national or governmental screening program, and participant age on the $I^{2}$ and pooled prevalence.

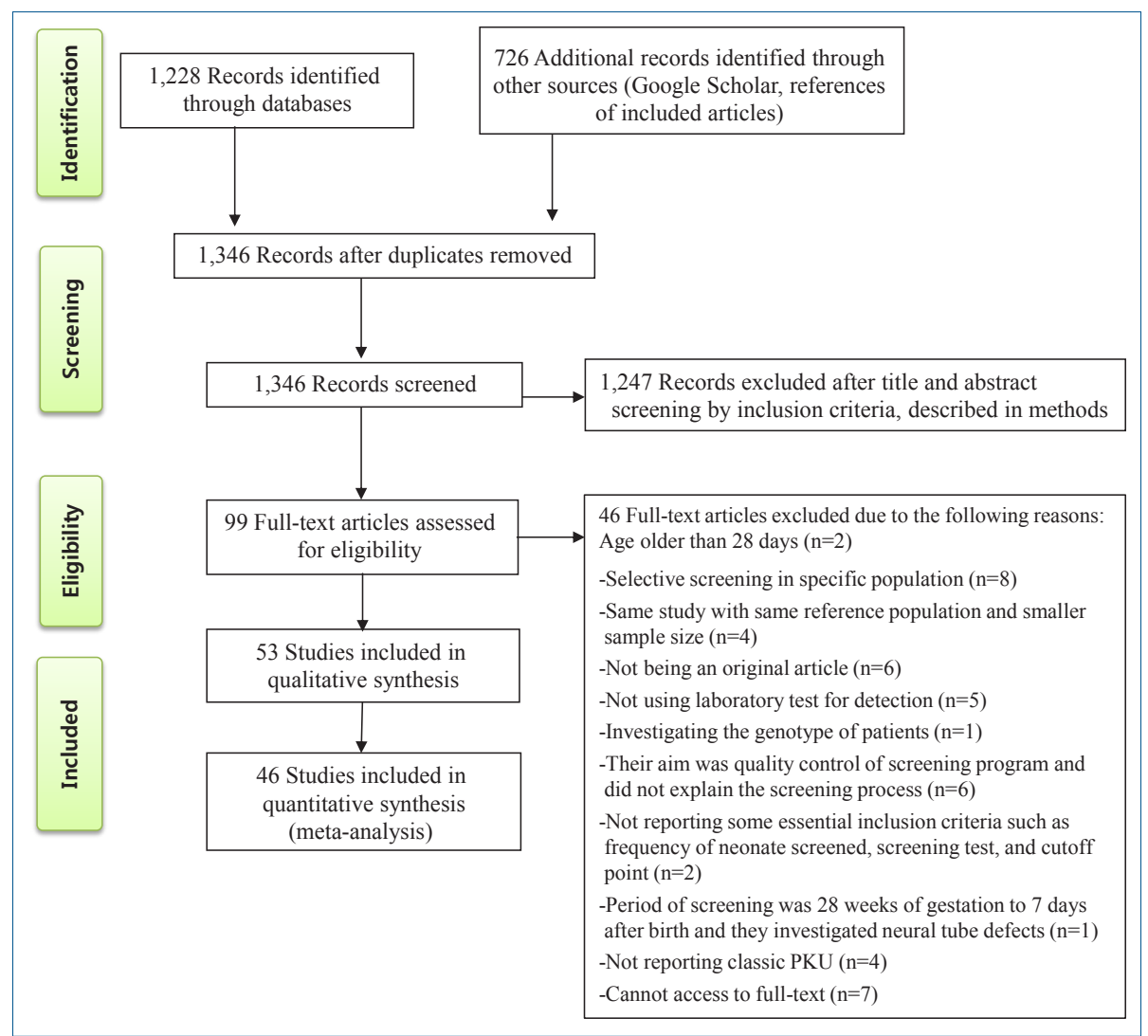

Fig. 1. Flow diagram of the literature search and study selection process. PKU, phenylketonuria. 


\section{Results}

\section{Study selection}

After a comprehensive search, 1,228 relevant articles were identified, 608 duplicates were removed. The relevance of the remaining 1,346 studies was evaluated based on the titles/ abstracts alone; of them, 99 studies were subjected to fulltext review, which eliminated another 46 studies according to the inclusion and exclusion criteria. Finally, 53 studies with $119,152,905$ participants were included in the present systematic review; of them, 46 were included in the meta-analysis (Fig. 1).

\section{Risk of bias}

In the bias assessment, 7 studies scored below 6 (high risk) and were excluded from the meta-analysis; thus, the pooled global prevalence was estimated without them. ${ }^{11-13,21-24)}$ Moreover, 33 of the studies scored 6-8 (moderate risk), ${ }^{1,9,16,17,25-53)}$ while the other 13 studies had low risk. ${ }^{2,10,54-64)}$

\section{Study characteristics}

In this section, the systematic review results are presented based on different characteristics, including region, test, and participant characteristics.

\section{Region characteristics}

The included studies (1964-2017) are presented in Table 1. The longest study, conducted in France, examined 35 years of screening data from 1966 to $2001 .^{56)}$ The largest screening population was in China $(35,795,550$ newborns for 30 years from the start of the screening project); the largest number of cases was detected (3,082 patients) in this study. ${ }^{60)}$ However, the smallest population belonged to a study conducted in Iraq in 2015 , and only 8,255 newborns were screened. ${ }^{13)}$

\section{Participants' characteristics}

Sampling age at screening was below 5 days in 30 studies, $1,2,9,10,16,17,21,23,27,29,31-33,35,39,41,43-46,48,50-53,56-58,60,61) 5-10$ days in 15 studies, ${ }^{13,22,28,30,34,37,42,47,49,54,55,59,62,63,65)}$ and over 10 days in 3 studies. ${ }^{25,26,38)}$ Five studies did not report newborn age at screening. ${ }^{11,12,24,36,64)}$

The neonatal participation rate was reported in 23 studies

Table 1. Description of studies included in the study

\begin{tabular}{|c|c|c|c|c|c|c|c|c|c|c|c|c|c|c|}
\hline ID & Study & $\begin{array}{l}\text { Study } \\
\text { location }\end{array}$ & $\begin{array}{l}\text { Population } \\
\text { size }\end{array}$ & $\begin{array}{l}\text { Study } \\
\text { period }\end{array}$ & $\begin{array}{l}\text { Age } \\
\text { taking } \\
\text { a blood } \\
\text { sample }\end{array}$ & $\begin{array}{l}\text { Screening test/ } \\
\text { cutoff level } \\
\text { (mg/dL) }\end{array}$ & $\begin{array}{c}\text { No. of } \\
\text { cases in } \\
\text { screening } \\
\text { test (inci- } \\
\text { dence per } \\
100,000 \text { ) }\end{array}$ & $\begin{array}{l}\text { Diagnostic test/ } \\
\text { cutoff level for } \\
\text { classic PKU } \\
\text { (mg/dL) }\end{array}$ & $\begin{array}{c}\text { No. of } \\
\text { classic } \\
\text { PKU cases } \\
\text { (incidence } \\
\text { per } \\
100,000 \text { ) }\end{array}$ & $\begin{array}{l}\text { Consan- } \\
\text { guinity }\end{array}$ & $\begin{array}{l}\text { Neo- } \\
\text { natal } \\
\text { partici- } \\
\text { pation } \\
\text { rate }\end{array}$ & $\begin{array}{c}\text { Rist } \\
\text { of } \\
\text { bias } \\
\text { Score }\end{array}$ & Remarks & $\begin{array}{l}\text { WHO } \\
\text { regions }\end{array}$ \\
\hline 1 & $\begin{array}{l}\text { MacCready, }{ }^{25)} \\
1964\end{array}$ & $\begin{array}{l}\text { USA/Massa- } \\
\text { chusetts }\end{array}$ & 134,580 & $\begin{array}{l}1962- \\
1964\end{array}$ & 28 Days & Guthrie/4 & NA & Guthrie/ $\geq 4$ & $14(10.4)$ & NA & NA & 7 & & $\begin{array}{l}\text { Pan Ameri- } \\
\text { can }\end{array}$ \\
\hline 2 & $\begin{array}{l}\text { Peterson, }{ }^{26)} \\
1968\end{array}$ & $\begin{array}{l}\text { USA/Califor- } \\
\text { nia }\end{array}$ & 311,953 & 1966 & 30 Days & $\begin{array}{l}\text { Guthrie and Fluorome- } \\
\text { tric/20 }\end{array}$ & NA & $\begin{array}{l}\text { Guthrie and Fluoro- } \\
\text { metric/ } \geq 20\end{array}$ & $16(5.12)$ & NA & NA & 6 & & $\begin{array}{l}\text { Pan Ameri- } \\
\text { can }\end{array}$ \\
\hline 3 & Fox, ${ }^{27)} 1971$ & $\begin{array}{l}\text { Canada/Ma- } \\
\text { nitoba }\end{array}$ & 85,868 & $\begin{array}{c}1966- \\
1970\end{array}$ & 4-5 Days & Guthrie test/20 & NA & Guthrie test $/ \geq 20$ & $5(5.82)$ & NA & $95.7 \%$ & 8 & & $\begin{array}{l}\text { Pan Ameri- } \\
\text { can }\end{array}$ \\
\hline 4 & Alm, ${ }^{54)} 1981$ & Sweden & $1,362,497$ & $\begin{array}{l}1965- \\
1979\end{array}$ & 4-6 Days & $\begin{array}{l}\text { Ion exchange chroma- } \\
\text { tography/4.12 }\end{array}$ & $\begin{array}{c}312 \\
(22.89)\end{array}$ & $\begin{array}{l}\text { Ion exchange chro- } \\
\text { matography/ } \geq 4.12\end{array}$ & $43(3.15)$ & NA & $86 \%$ & 9 & $\begin{array}{l}\text { The phenylalanine cut } \\
\text { point reported } 0.25 \\
\mathrm{mmol} / \mathrm{L} \text {, this is equal } \\
\text { to } 4.12 \mathrm{mg} / \mathrm{dL}\end{array}$ & Europe \\
\hline 5 & $\begin{array}{l}\text { Antonozzi, } \\
1982\end{array}$ & $\begin{array}{l}\text { Italy } / 3 \text { regi- } \\
\text { ons }\end{array}$ & 220,000 & $\begin{array}{l}1974- \\
1981\end{array}$ & $\begin{array}{l}\text { Median of } \\
7.6 \text { days }\end{array}$ & $\begin{array}{l}\text { Ion exchange chroma- } \\
\text { tography/1.65 }\end{array}$ & $\begin{array}{c}211 \\
(95.9)\end{array}$ & $\begin{array}{l}\text { Ion exchange chro- } \\
\text { matography/ } \geq 1.65\end{array}$ & $23(10.45)$ & NA & $42 \%$ & 6 & $\begin{array}{l}\text { The phenyl alanine cut } \\
\text { point reported } 100 \\
\mu \mathrm{mol} / \mathrm{dL}, \text { this is equal } \\
\text { to } 1.65 \mathrm{mg} / \mathrm{dL}\end{array}$ & Europe \\
\hline 6 & $\begin{array}{l}\text { Farhud, }_{,}^{22)} \\
1982\end{array}$ & Iran/Tehran & 8,633 & 1982 & 4-8 Days & Guthrie/20 & NA & Guthrie/ $\geq 20$ & $1(11.58)$ & NA & NA & 5 & & $\begin{array}{r}\text { Eastern Me- } \\
\text { diterranean }\end{array}$ \\
\hline 7 & Liu, $^{29)} 1986$ & $\begin{array}{l}\text { china/11 } \\
\text { province }\end{array}$ & 198,320 & $\begin{array}{c}1982- \\
1985\end{array}$ & 1-3 Days & Guthrie/4 & $\begin{array}{c}225 \\
(113.4)\end{array}$ & Guthrie/ $\geq 15$ & $9(4.53)$ & NA & NA & 8 & $\begin{array}{l}\text { Reports overall inci- } \\
\text { dence } 1: 16500 \text { but } \\
\text { the incidence is } 1: \\
22035\end{array}$ & $\begin{array}{l}\text { Western } \\
\text { Pacific }\end{array}$ \\
\hline 8 & $\begin{array}{l}\text { Mathias, }{ }_{r}^{30)} \\
1986\end{array}$ & $\begin{array}{l}\text { West Ger- } \\
\text { many }\end{array}$ & 940,369 & $\begin{array}{r}1969- \\
1984\end{array}$ & 5-7 Days & Guthrie/15 & $\begin{array}{c}170 \\
(18.07)\end{array}$ & $\begin{array}{l}\text { Ion exchange chro- } \\
\text { matography/ } \geq 15\end{array}$ & $94(10)$ & NA & NA & 8 & & Europe \\
\hline 9 & Özalp, ${ }^{31)} 1986$ & $\begin{array}{l}\text { Turkey/An- } \\
\text { kara }\end{array}$ & 20,979 & $\begin{array}{l}1983- \\
1985\end{array}$ & $>24 \mathrm{Hr}$ & Guthrie/4 & NA & Fluorometric/ $\geq 20$ & $8(38.13)$ & NA & NA & 8 & & Europe \\
\hline 10 & Aoki, ${ }^{55)} 1988$ & Japan & $12,168,645$ & $\begin{array}{c}1977- \\
1985\end{array}$ & 5-7 Days & Guthrie/20 & NA & Guthrie/ $\geq 20$ & $102(0.83)$ & NA & $87 \%$ & 10 & & $\begin{array}{l}\text { Western } \\
\text { Pacific }\end{array}$ \\
\hline 11 & $\begin{array}{l}\text { Missiou- } \\
\text { Tsagaraki, }{ }^{22)} \\
1988\end{array}$ & Greece & $1,042,000$ & $\begin{array}{c}1974- \\
1986\end{array}$ & $>24 \mathrm{Hr}$ & $\begin{array}{l}\text { Thin-layer chromato- } \\
\text { graphy/4 }\end{array}$ & $\begin{array}{c}776 \\
(74.47)\end{array}$ & $\begin{array}{l}\text { Thin-layer chroma- } \\
\text { tography/ } \geq 20\end{array}$ & $43(4.12)$ & NA & NA & 8 & & Europe \\
\hline 12 & Chen, ${ }^{33)} 1989$ & $\begin{array}{l}\text { China/Shang- } \\
\text { hai }\end{array}$ & 358,767 & $\begin{array}{l}1981- \\
1989\end{array}$ & >3 Days & Guthrie/4 & $\begin{array}{c}72 \\
(20.06)\end{array}$ & Fluorometric/ $\geq 20$ & $21(5.85)$ & NA & $33 \%$ & 6 & & $\begin{array}{l}\text { Western Pa- } \\
\text { cific }\end{array}$ \\
\hline 13 & Smith, ${ }^{34)} 1991$ & $\begin{array}{l}\text { United King- } \\
\text { dom }\end{array}$ & $3,796,645$ & $\begin{array}{l}1984- \\
1988\end{array}$ & 6-7 Days & $\begin{array}{l}9 \text { Laboratories Guthrie, } \\
6 \text { used fluorometric } \\
\text { and } 11 \text { used thin layer } \\
\text { or paper chromato- } \\
\text { graphy/4 }\end{array}$ & NA & $\begin{array}{l}9 \text { Laboratories Guth- } \\
\text { rie, } 6 \text { used fluoro- } \\
\text { metric and } 11 \text { used } \\
\text { layer or paper chro- } \\
\text { matography/ } \geq 20\end{array}$ & $273(7.19)$ & & $100 \%$ & 8 & $\begin{array}{l}\text { The phenylalanine cut } \\
\text { point reported } 240 \\
\mu \mathrm{mol} / \mathrm{dL} \text {, this is equal } \\
\text { to } 4 \mathrm{mg} / \mathrm{dL}\end{array}$ & \\
\hline 14 & $\begin{array}{l}\text { Gerasimova, } \\
\text { 35) } 1992\end{array}$ & $\begin{array}{l}\text { Russia, Mos- } \\
\text { cow }\end{array}$ & 139,664 & $\begin{array}{c}1990- \\
1991\end{array}$ & 4-5 Days & Fluorometric/3 & $\begin{array}{c}529 \\
(378)\end{array}$ & Fluorometric/ $\geq 20$ & $21(15.03)$ & NA & NA & 7 & $\begin{array}{l}\text { The phenylalanine cut } \\
\text { point reported } 180 \\
\mu \mathrm{mol} / \mathrm{dL} \text {, this is equal } \\
\text { to } 3 \mathrm{mg} / \mathrm{dL}\end{array}$ & \\
\hline
\end{tabular}




\begin{tabular}{|c|c|c|c|c|c|c|c|c|c|c|c|c|c|c|}
\hline ID & Study & $\begin{array}{l}\text { Study } \\
\text { location }\end{array}$ & $\begin{array}{l}\text { Population } \\
\text { size }\end{array}$ & $\begin{array}{l}\text { Study } \\
\text { period }\end{array}$ & $\begin{array}{l}\text { Age } \\
\text { taking } \\
\text { a blood } \\
\text { sample }\end{array}$ & $\begin{array}{l}\text { Screening test/ } \\
\text { cutoff level } \\
\text { (mg/dL) }\end{array}$ & $\begin{array}{c}\text { No. of } \\
\text { cases in } \\
\text { screening } \\
\text { test (inci- } \\
\text { dence per } \\
100,000 \text { ) }\end{array}$ & $\begin{array}{l}\text { Diagnostic test/ } \\
\text { cutoff level for } \\
\text { classic PKU } \\
\text { (mg/dL) }\end{array}$ & $\begin{array}{c}\text { No. of } \\
\text { classic PKU } \\
\text { cases (inci- } \\
\text { dence per } \\
100,000 \text { ) }\end{array}$ & $\begin{array}{l}\text { Consan- } \\
\text { guinity }\end{array}$ & $\begin{array}{l}\text { Neo- } \\
\text { natal } \\
\text { partici- } \\
\text { pation } \\
\text { rate }\end{array}$ & $\begin{array}{c}\text { Rist } \\
\text { of } \\
\text { bias } \\
\text { Score }\end{array}$ & Remarks & $\begin{array}{l}\text { WHO } \\
\text { regions }\end{array}$ \\
\hline 15 & $\begin{array}{l}\text { Cabalska, } \\
1993\end{array}$ & Poland & $2,861,504$ & $\begin{array}{r}1965- \\
1990\end{array}$ & NA & Guthrie/4 & NA & Guthrie/ $\geq 20$ & $368(12.86)$ & NA & $\begin{array}{l}40 \%- \\
100 \%\end{array}$ & 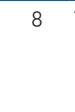 & $\begin{array}{l}\text { They just presented data } \\
\text { from the National Research } \\
\text { Institute for Mother and } \\
\text { Child }\end{array}$ & Europe \\
\hline 16 & $\begin{array}{l}\text { Fernandez- } \\
\text { Iglesias, }^{37)} \\
1995\end{array}$ & $\begin{array}{l}\text { Spain/Princi- } \\
\text { pado }\end{array}$ & 75,488 & $\begin{array}{l}1982- \\
1993\end{array}$ & 5-8 Days & $\begin{array}{l}\text { Thin-layer chro- } \\
\text { matography/4 }\end{array}$ & NA & $\begin{array}{l}\text { High performance } \\
\text { liquid chromatogra- } \\
\text { phy/ } \geq 4\end{array}$ & $5(6.62)$ & NA & NA & 7 & $\begin{array}{l}\text { The phenylalanine cut point } \\
\text { reported } 240 \mu \mathrm{mol} / \mathrm{dL} \text {, this } \\
\text { is equal to } 4 \mathrm{mg} / \mathrm{dL}\end{array}$ & Europe \\
\hline 17 & $\begin{array}{l}\text { Hitzeroth, } \\
1995\end{array}$ & $\begin{array}{l}\text { South Africa/ } \\
\text { Pretoria }\end{array}$ & 59,600 & $\begin{array}{l}1979- \\
1986\end{array}$ & 3-5 Days & $\begin{array}{l}\text { Thin-layer chro- } \\
\text { matography/NA }\end{array}$ & NA & $\begin{array}{l}\text { Thin-layer chroma- } \\
\text { tography/NA }\end{array}$ & $1(1.67)$ & NA & NA & 5 & & Africa \\
\hline 18 & $\begin{array}{l}\text { Özalp, } \\
1995\end{array}$ & Turkey & 576,122 & $\begin{array}{l}1987- \\
1994\end{array}$ & $>24 \mathrm{Hr}$ & Fluorometric/20 & NA & Fluorometric/ $\geq 20$ & $96(16.66)$ & $45 \%$ & NA & 7 & $\begin{array}{l}\text { The phenylalanine cut point } \\
\text { reported } 1,200 \mu \mathrm{mol} / \mathrm{dL} \text {, } \\
\text { this is equal to } 20 \mathrm{mg} / \mathrm{dL} \text { ) }\end{array}$ & Europe \\
\hline 19 & $\begin{array}{l}\text { Kucinskas, }{ }^{38)} \\
1996\end{array}$ & Lithuania & 907,168 & $\begin{array}{l}1975- \\
1993\end{array}$ & 21 Days & Fluorometric/2.5 & NA & Fluorometric/ $\geq 2.5$ & $85(9.36)$ & NA & NA & 8 & $\begin{array}{l}\text { The phenylalanine cut point } \\
\text { reported } 150 \mu \mathrm{mol} / \mathrm{dL} \text {, this } \\
\text { is equal to } 2.5 \mathrm{mg} / \mathrm{dL}\end{array}$ & Europe \\
\hline 20 & $\begin{array}{l}\text { Ounap }^{66)} \\
1998\end{array}$ & Estonia & 36,074 & $\begin{array}{l}1993- \\
1995\end{array}$ & 3-5 Days & Fluorometric/3 & NA & Fluorometric $/ \geq 3$ & $6(16.63)$ & NA & $85 \%$ & 7 & $\begin{array}{l}\text { The phenyl alanine cut point } \\
\text { reported } 180 \mu \mathrm{mol} / \mathrm{dL} \text {, this } \\
\text { is equal to } 3 \mathrm{mg} / \mathrm{dL}\end{array}$ & Europe \\
\hline 21 & $\begin{array}{l}\text { Abadie, }^{56)} \\
2001\end{array}$ & France & $21,500,000$ & $\begin{array}{l}1966- \\
2001\end{array}$ & 3-5 Days & $\begin{array}{l}\text { Until } 1990 \text { (Guth- } \\
\text { rie) } 1991-2001 \\
\text { (Fluorometric)/10 }\end{array}$ & $\begin{array}{l}1,426 \\
(6.63)\end{array}$ & $\begin{array}{l}\text { Until } 1990 \text { (Guthrie), } \\
1991-2001 \text { (Fluo- } \\
\text { rometric)/ } \geq 10\end{array}$ & $1,164(5.41)$ & NA & $\begin{array}{l}3 \%- \\
65 \%\end{array}$ & 9 & $\begin{array}{l}\text { The phenylalanine cut point } \\
\text { reported } 600 \mu \mathrm{mol} / \mathrm{dL} \text {, this } \\
\text { is equal to } 10 \mathrm{mg} / \mathrm{dL}\end{array}$ & Europe \\
\hline 22 & $\begin{array}{l}\text { Zytkovicz, }^{39)} \\
2001\end{array}$ & England & 257,000 & $\begin{array}{l}1999- \\
2001\end{array}$ & 1-3 Days & MS/MS/2.29 & $\begin{array}{c}92 \\
(35.79)\end{array}$ & $\mathrm{MS} / \mathrm{MS} / \geq 2.29$ & $7(2.72)$ & NA & NA & 8 & $\begin{array}{l}\text { The phenylalanine cut point } \\
\text { reported } 139 \mu \mathrm{mol} / \mathrm{dL} \text {, this } \\
\text { is equal to } 2.29 \mathrm{mg} / \mathrm{dL}\end{array}$ & Europe \\
\hline 23 & $\begin{array}{l}\text { Schulze, } \\
2002\end{array}$ & Germany & 423,773 & $\begin{array}{l}1994- \\
1999\end{array}$ & $\begin{array}{l}5 \text { Days } \\
\text { (1-10 } \\
\text { days })\end{array}$ & MS/MS/2.5 & NA & $\mathrm{MS} / \mathrm{MS} / \geq 10$ & $41(9.67)$ & NA & NA & 8 & $\begin{array}{l}\text { The phenylalanine cut point } \\
\text { reported } 150 \mu \mathrm{mol} / \mathrm{dL} \text {, this } \\
\text { is equal to } 2.5 \mathrm{mg} / \mathrm{dl}\end{array}$ & Europe \\
\hline 24 & $\begin{array}{l}\text { Zaffanello, } \\
2002\end{array}$ & $\begin{array}{l}\text { Northeastern } \\
\text { Italy }\end{array}$ & $1,142,338$ & $\begin{array}{l}1978- \\
1997\end{array}$ & 3-5 Days & Guthrie/2 & NA & $\begin{array}{l}\text { High performance } \\
\text { liquid chromatogra- } \\
\text { phy/ } \geq 20\end{array}$ & $25(2.18)$ & NA & $97 \%$ & 9 & & Europe \\
\hline 25 & $\begin{array}{l}\text { Capistrano- } \\
\text { Estrada, } \\
2003\end{array}$ & Philippines & 189,720 & $\begin{array}{l}1996- \\
2001\end{array}$ & $>24 \mathrm{Hr}$ & Guthrie/3.3 & $\begin{array}{c}75 \\
(39.53)\end{array}$ & Guthrie/ $\geq 3.3$ & $3(1.58)$ & NA & NA & 6 & $\begin{array}{l}\text { The phenylalanine cut point } \\
\text { reported } 200 \mu \mathrm{mol} / \mathrm{dL} \text {, this } \\
\text { is equal to } 3.3 \mathrm{mg} / \mathrm{dL}\end{array}$ & $\begin{array}{l}\text { Western Pa- } \\
\text { cific }\end{array}$ \\
\hline 26 & $\begin{array}{l}\text { Charoensiri- } \\
\text { watana }^{42)} \\
2003\end{array}$ & Thailand & $1,425,025$ & $\begin{array}{l}1992- \\
2001\end{array}$ & 2-7 Days & Guthrie/4 & $\begin{array}{c}321 \\
(22.52)\end{array}$ & Fluorometric/ $\geq 4$ & $5(0.35)$ & NA & NA & 7 & & $\begin{array}{l}\text { South-East } \\
\text { Asia }\end{array}$ \\
\hline 27 & $\begin{array}{l}\text { Jiang, }^{43)} \\
2003\end{array}$ & $\begin{array}{l}\text { China/Guang- } \\
\text { dong }\end{array}$ & 461,805 & NA & 3 Days & $\begin{array}{l}\text { Guthrie and Fluo- } \\
\text { rometric/2 }\end{array}$ & NA & $\begin{array}{l}\text { Guthrie and Fluoro- } \\
\text { metric } / \geq 20\end{array}$ & $6(1.29)$ & NA & NA & 7 & $\begin{array}{l}\text { The phenylalanine cut point } \\
\text { reported } 120 \mu \mathrm{mol} / \mathrm{dL} \text {, this } \\
\text { is equal to } 2 \mathrm{mg} / \mathrm{dL}\end{array}$ & $\begin{array}{l}\text { Western Pa- } \\
\text { cific }\end{array}$ \\
\hline 28 & $\begin{array}{l}\text { Yoon, }{ }^{44)} \\
2005\end{array}$ & South Korea & $5,243,841$ & $\begin{array}{l}1996- \\
2006\end{array}$ & NA & $\begin{array}{l}\text { Guthrie and Fluo- } \\
\text { rometric/4 }\end{array}$ & NA & $\begin{array}{l}\text { High performance } \\
\text { liquid chromatogra- } \\
\text { phy } / \geq 20\end{array}$ & $16(0.3)$ & NA & NA & 8 & & $\begin{array}{l}\text { Western Pa- } \\
\text { cific }\end{array}$ \\
\hline 29 & $\begin{array}{l}\text { Pangkanon, } \\
\text { 67) } 2009\end{array}$ & Thailand & 79,179 & $\begin{array}{l}2001- \\
2004\end{array}$ & 2-3 Days & MS/MS/2.29 & NA & $\mathrm{MS} / \mathrm{MS} / \geq 20$ & $5(6.31)$ & NA & $5.40 \%$ & 9 & $\begin{array}{l}\text { The phenylalanine cut point } \\
\text { reported } 139 \mu \mathrm{mol} / \mathrm{dL} \text {, this } \\
\text { is equal to } 2.29 \mathrm{mg} / \mathrm{dL}\end{array}$ & $\begin{array}{l}\text { South-East } \\
\text { Asia }\end{array}$ \\
\hline 30 & $\begin{array}{l}\text { Senemar, } \\
2009\end{array}$ & Iran/Fars & 70,477 & $\begin{array}{l}2000- \\
2005\end{array}$ & 3 Days & Fluorometric/4 & NA & Fluorometric/ $\geq 4$ & $15(21.28)$ & $86.60 \%$ & NA & 7 & & $\begin{array}{r}\text { Eastern Me- } \\
\text { diterranean }\end{array}$ \\
\hline 31 & $\begin{array}{l}\text { Cornejo, } \\
2010\end{array}$ & Chile & $2,478,123$ & $\begin{array}{l}1992- \\
2008\end{array}$ & 3.6 Mean & Fluorometric/20 & NA & $\begin{array}{l}\text { 1998-2002 Fluoro- } \\
\text { metric-2002-2008 } \\
\text { MS/MS/ } \geq 20\end{array}$ & $131(5.28)$ & NA & $\begin{array}{l}48- \\
98 \%\end{array}$ & 9 & & $\begin{array}{l}\text { Pan Ameri- } \\
\text { can }\end{array}$ \\
\hline 32 & $\begin{array}{l}\text { Habib }_{,}^{45)} \\
2010\end{array}$ & Iran /Fars & 175,235 & $\begin{array}{l}2004- \\
2007\end{array}$ & 3-5 Days & $\begin{array}{l}\text { Enzymatic colori- } \\
\text { metric method } / 4\end{array}$ & $\begin{array}{c}30 \\
(17.11)\end{array}$ & $\begin{array}{l}\text { High performance } \\
\text { liquid chromatogra- } \\
\text { phy/ } \geq 10\end{array}$ & $28(15.97)$ & NA & NA & 8 & & $\begin{array}{r}\text { Eastern Me- } \\
\text { diterranean }\end{array}$ \\
\hline 33 & $\begin{array}{l}\text { Karamifar, } \\
2010\end{array}$ & Iran /Fars & 76,966 & $\begin{array}{l}2007- \\
2008\end{array}$ & 3-5 Days & $\begin{array}{l}\text { Enzymatic colori- } \\
\text { metric method/2 }\end{array}$ & $\begin{array}{c}9 \\
(11.69)\end{array}$ & $\begin{array}{l}\text { High performance } \\
\text { liquid chromatogra- } \\
\text { phy } / \geq 20\end{array}$ & $8(10.39)$ & NA & NA & 8 & & $\begin{array}{r}\text { Eastern Me- } \\
\text { diterranean }\end{array}$ \\
\hline 34 & $\mathrm{Niu}^{46)} 2010$ & Taiwan & $1,495,132$ & $\begin{array}{l}2000- \\
2009\end{array}$ & 2-3 Days & $\mathrm{MS} / \mathrm{MS} / 4$ & NA & $\mathrm{MS} / \mathrm{MS} / \geq 20$ & $5(0.33)$ & NA & $>99 \%$ & 8 & $\begin{array}{l}\text { The phenylalanine cut point } \\
\text { reported } 240 \mu \mathrm{mol} / \mathrm{dL} \text {, this } \\
\text { is equal to } 4 \mathrm{mg} / \mathrm{dL}\end{array}$ & $\begin{array}{l}\text { Western Pa- } \\
\text { cific }\end{array}$ \\
\hline 35 & $\begin{array}{l}\text { Vilarinho, }^{59)} \\
2010\end{array}$ & Portugal & 316,243 & $\begin{array}{l}2004- \\
2008\end{array}$ & 3-6 Days & $\mathrm{MS} / \mathrm{MS} / 2.5$ & NA & $\mathrm{MS} / \mathrm{MS} / \geq 6$ & $26(8.22)$ & NA & $99.80 \%$ & 9 & $\begin{array}{l}\text { The phenylalanine cut point } \\
\text { reported } 150 \mu \mathrm{mol} / \mathrm{dL} \text {, this } \\
\text { is equal to } 2.5 \mathrm{mg} / \mathrm{dL}\end{array}$ & Europe \\
\hline 36 & $\begin{array}{l}\text { Sutivijit,47) } \\
2011\end{array}$ & $\begin{array}{l}\text { Thailand/Sou- } \\
\text { thern Region }\end{array}$ & $1,118,676$ & $\begin{array}{l}2000- \\
2009\end{array}$ & $>2$ Days & Guthrie/4 & $\begin{array}{c}120 \\
(10.72)\end{array}$ & Fluorometric/ $\geq 4$ & $5(0.44)$ & NA & $\begin{array}{l}\text { near } \\
100 \%\end{array}$ & 8 & & $\begin{array}{l}\text { South-East } \\
\text { Asia }\end{array}$ \\
\hline 37 & $\begin{array}{l}\text { Botler, }^{10)} \\
2012\end{array}$ & Brazil & 541,248 & $\begin{array}{l}2005- \\
2007\end{array}$ & 2-5 Days & Fluorometric/4 & $\begin{array}{c}64 \\
(11.82)\end{array}$ & $\begin{array}{l}\text { Thin layer amino acid } \\
\text { chromatography } \geq \\
10\end{array}$ & $26(4.8)$ & NA & $\begin{array}{l}71- \\
80 \%\end{array}$ & 9 & & $\begin{array}{l}\text { Pan Ameri- } \\
\text { can }\end{array}$ \\
\hline 38 & $\mathrm{Shi}^{60)} 2012$ & China & $35,795,550$ & $\begin{array}{l}1981- \\
2011\end{array}$ & 2-3 Days & Guthrie/2 & NA & $\begin{array}{l}\text { Fluorometric: } \geq 2 \\
\text { Guthrie: } \geq 4\end{array}$ & $3,082(8.6)$ & NA & $\begin{array}{c}3.86 \% \\
\text { in } 2003 \\
\text { and } \\
59.01 \% \\
\text { in } 2009\end{array}$ & 9 & $\begin{array}{l}\text { The phenylalanine cut point } \\
\text { reported } 120 \mu \mathrm{mol} / \mathrm{dL} \text {, } \\
\text { this is equal to } 2 \mathrm{mg} / \mathrm{dL}\end{array}$ & $\begin{array}{l}\text { Western Pa- } \\
\text { cific }\end{array}$ \\
\hline 39 & $\begin{array}{l}\text { Yang, }_{1}^{611} \\
2012\end{array}$ & $\begin{array}{l}\text { China/Zheji- } \\
\text { ang }\end{array}$ & $3,791,538$ & $\begin{array}{l}1999- \\
2010\end{array}$ & 3-5 Days & $\begin{array}{l}\text { Fluorescent ninhy- } \\
\text { drine method/2 }\end{array}$ & NA & $\begin{array}{l}\text { Fluorescent ninhy- } \\
\text { drine method/ } \geq 2\end{array}$ & $143(3.77)$ & NA & NA & 9 & $\begin{array}{l}\text { The phenylalanine cut point } \\
\text { reported } 120 \mu \mathrm{mol} / \mathrm{dL} \text {, } \\
\text { this is equal to } 2 \mathrm{mg} / \mathrm{dL}\end{array}$ & $\begin{array}{l}\text { Western Pa- } \\
\text { cific }\end{array}$ \\
\hline
\end{tabular}


Table 1. Description of studies included in the study (Continued)

\begin{tabular}{|c|c|c|c|c|c|c|c|c|c|c|c|c|c|c|}
\hline ID & Study & $\begin{array}{c}\text { Study } \\
\text { location }\end{array}$ & $\begin{array}{l}\text { Population } \\
\text { size }\end{array}$ & $\begin{array}{l}\text { Study } \\
\text { period }\end{array}$ & $\begin{array}{l}\text { Age } \\
\text { taking } \\
\text { a blood } \\
\text { sample }\end{array}$ & $\begin{array}{l}\text { Screening test/ } \\
\text { cutoff level } \\
\text { (mg/dL) }\end{array}$ & $\begin{array}{l}\text { No. of } \\
\text { cases in } \\
\text { screening } \\
\text { test (inci- } \\
\text { dence per } \\
100,000 \text { ) }\end{array}$ & $\begin{array}{l}\text { Diagnostic test/ } \\
\text { cutoff level for } \\
\text { classic PKU } \\
\text { (mg/dL) }\end{array}$ & $\begin{array}{l}\text { No. of } \\
\text { classic PKU } \\
\text { cases (inci- } \\
\text { dence per } \\
100,000 \text { ) }\end{array}$ & $\begin{array}{l}\text { Consan- } \\
\text { guinity }\end{array}$ & $\begin{array}{l}\text { Neo- } \\
\text { natal } \\
\text { partici- } \\
\text { pation } \\
\text { rate }\end{array}$ & $\begin{array}{c}\text { Rist } \\
\text { of } \\
\text { bias } \\
\text { Score }\end{array}$ & Remarks & $\begin{array}{l}\text { WHO } \\
\text { regions }\end{array}$ \\
\hline 40 & $\begin{array}{l}\text { Dluholucký, }{ }^{11)} \\
2013\end{array}$ & Slovakia & 927,524 & $\begin{array}{r}1995- \\
2012\end{array}$ & NA & Guthrie/NA & NA & Fluorometric (NA) & $157(16.92)$ & NA & $98 \%$ & 5 & & Europe \\
\hline 41 & $\begin{array}{l}\text { Al Hosani, } \\
2014\end{array}$ & $\begin{array}{l}\text { United Arab } \\
\text { Emirates }\end{array}$ & 750,365 & $\begin{array}{l}1995- \\
2011\end{array}$ & $>2$ Days & $\begin{array}{l}\text { Time-resolved } \\
\text { fluorescence/4 }\end{array}$ & $\begin{array}{c}57 \\
(7.59)\end{array}$ & $\begin{array}{l}\text { 1995-2001 time-re- } \\
\text { solved fluorescence } \\
\text { application } 2011 \\
\text { MS/MS/ } \geq 20\end{array}$ & $51(6.79)$ & NA & $\begin{array}{c}1995 \\
-50 \% \\
2010- \\
95 \%\end{array}$ & 10 & & $\begin{array}{r}\text { Eastern } \mathrm{Me}- \\
\text { diterranean }\end{array}$ \\
\hline 42 & $\begin{array}{l}\text { Dluholucký, } \\
2014\end{array}$ & Slovakia & 82,892 & $\begin{array}{l}2013- \\
2014\end{array}$ & NA & $\mathrm{MS} / \mathrm{MS} / \mathrm{NA}$ & NA & MS/MS (NA) & $5(6.03)$ & NA & NA & 5 & & Europe \\
\hline 43 & $\begin{array}{l}\text { Ramalho, } \\
2014\end{array}$ & $\begin{array}{l}\text { Brazil/Ser- } \\
\text { gipe }\end{array}$ & 43,449 & $\begin{array}{l}2007- \\
2008\end{array}$ & 2-6 Days & $\begin{array}{l}\text { Enzymatic colori- } \\
\text { metric method/ } \\
5\end{array}$ & NA & $\begin{array}{l}\text { Enzymatic colorime- } \\
\text { tric method } / \geq 20\end{array}$ & $4(9.2)$ & NA & $78.93 \%$ & 9 & & $\begin{array}{l}\text { Pan Ameri- } \\
\text { can }\end{array}$ \\
\hline 44 & $\begin{array}{l}\text { Hamawandi, }{ }^{13)} \\
2015\end{array}$ & $\begin{array}{l}\text { Iraq/Sulai- } \\
\text { mani }\end{array}$ & 8,255 & $\begin{array}{l}2013- \\
2014\end{array}$ & 3-10 Days & ELISA/4 & $\begin{array}{c}11 \\
(133.25)\end{array}$ & $\begin{array}{l}\text { High performance } \\
\text { liquid chromatogra- } \\
\text { phy/ } / \geq 4\end{array}$ & $1(12.11)$ & $100 \%$ & NA & 5 & & $\begin{array}{r}\text { Eastern } \mathrm{Me}- \\
\text { diterranean }\end{array}$ \\
\hline 45 & Šmon, ${ }^{21} 2015$ & Slovenia & 385,831 & $\begin{array}{l}1993- \\
2012\end{array}$ & 3-5 Days & Fluorometric/3.3 & NA & Fluorometric/ $\geq 20$ & $38(9.84)$ & NA & NA & 9 & $\begin{array}{l}\text { The phenylalanine cut point } \\
\text { reported } 0.2 \mathrm{mmol} / \mathrm{l} \text {, this } \\
\text { is equal to } 3.3 \mathrm{mg} / \mathrm{dL}\end{array}$ & Europe \\
\hline 46 & $\begin{array}{l}\text { Hassan, }^{49)} \\
2016\end{array}$ & Egypt & 25,276 & 2008 & 3-7 Days & $\mathrm{MS} / \mathrm{MS} / 2.5$ & NA & $\mathrm{MS} / \mathrm{MS} / \geq 1.69$ & $5(19.78)$ & NA & NA & 6 & $\begin{array}{l}\text { The phenylalanine cut point } \\
\text { reported } 150 \mu \mathrm{mol} / \mathrm{dL} \text {, } \\
\text { this is equal to } 2.5 \mathrm{mg} / \mathrm{dL}\end{array}$ & $\begin{array}{r}\text { Eastern Me- } \\
\text { diterranean }\end{array}$ \\
\hline 47 & $\begin{array}{l}\text { Zhong, }^{24)} \\
2016\end{array}$ & China & $13,187,196$ & 2013 & NA & NA/NA & NA & NA & $1,123(8.51)$ & NA & $\begin{array}{l}10 \%- \\
85 \%\end{array}$ & 4 & & $\begin{array}{l}\text { Western Pa- } \\
\text { cific }\end{array}$ \\
\hline 48 & $\begin{array}{l}\text { Al-Jasmi, }{ }^{21)} \\
2016\end{array}$ & $\begin{array}{l}\text { United Arab } \\
\text { Emirates }\end{array}$ & 136,049 & $\begin{array}{l}2011- \\
2014\end{array}$ & 3-5 Days & $\mathrm{MS} / \mathrm{MS} / \mathrm{NA}$ & NA & MS/MS (NA) & 11 (8.08) & $81 \%$ & NA & 5 & & $\begin{array}{r}\text { Eastern Me- } \\
\text { diterranean }\end{array}$ \\
\hline 49 & $\begin{array}{l}\text { Alkhazrajy, } \\
2016\end{array}$ & $\begin{array}{l}\text { Iraq/Bagh- } \\
\text { dad }\end{array}$ & 80,409 & 2014 & 3-5 Days & $\mathrm{MS} / \mathrm{MS} / 2.5$ & NA & $\mathrm{MS} / \mathrm{MS} / \geq 1.69$ & $6(7.46)$ & NA & $66 \%$ & 6 & $\begin{array}{l}\text { Self-calculated Prevalence. } \\
\text { Article did not report pre- } \\
\text { valence of PKU. } \\
\text { The phenylalanine cut point } \\
\text { reported } 150 \mu \mathrm{mol} / \mathrm{dL} \text {, this } \\
\text { is equal to } 2.5 \mathrm{mg} / \mathrm{dL}\end{array}$ & Eastern Me- \\
\hline 50 & $\begin{array}{l}\text { Saadatpour, }{ }^{50)} \\
2016\end{array}$ & $\begin{array}{l}\text { Iran/Hormo- } \\
\text { gan }\end{array}$ & 71,677 & $\begin{array}{l}2014- \\
2016\end{array}$ & 3-5 Days & ELISA/2 & $\begin{array}{c}15 \\
20.92)\end{array}$ & $\begin{array}{l}\text { High performance } \\
\text { liquid chromatogra- } \\
\text { phy } \geq 4 \mathrm{mg} / \mathrm{dL}\end{array}$ & $3(4.18)$ & $66 \%$ & $88 \%$ & 8 & $\begin{array}{l}\text { Consanguinity Reported } 53 \\
\% \text { but from } 3 \text { positive case } \\
2 \text { had Consanguinity mar- } \\
\text { riage and should correct } \\
66 \%\end{array}$ & $\begin{array}{l}\text { Eastern Me- } \\
\text { diterranean }\end{array}$ \\
\hline 51 & $\begin{array}{l}\text { Alfadhel, } \\
2017\end{array}$ & Saudi Arabia & 775,000 & $\begin{array}{l}2005- \\
2012\end{array}$ & $\begin{array}{l}\text { After } 24 \\
\text { hr of birth }\end{array}$ & $\mathrm{MS} / \mathrm{MS} / 3$ & NA & $M S / M S \geq 2.03$ & $53(6.83)$ & NA & NA & 7 & & $\begin{array}{l}\text { Eastern Me- } \\
\text { diterranean }\end{array}$ \\
\hline 52 & $\begin{array}{l}\text { Abbaskhanian, } \\
\text { 51) } 2017\end{array}$ & $\begin{array}{l}\text { Iran/Mazan- } \\
\text { daran }\end{array}$ & 407,244 & $\begin{array}{l}2007- \\
2015\end{array}$ & 3-5 Days & ELISA/4 & $\begin{array}{c}465 \\
(114.18)\end{array}$ & $\begin{array}{l}\text { High performance } \\
\text { liquid chromatogra- } \\
\text { phy>20 }\end{array}$ & $6(1.47)$ & NA & NA & 8 & & $\begin{array}{r}\text { Eastern Me- } \\
\text { diterranean }\end{array}$ \\
\hline 53 & $\begin{array}{l}\text { Motamedi, } \\
2017\end{array}$ & $\begin{array}{l}\text { Iran/Lores- } \\
\tan \end{array}$ & 384,993 & $\begin{array}{l}2006- \\
2016\end{array}$ & 3-5 Days & $\begin{array}{l}\text { High performance } \\
\text { liquid chromato- } \\
\text { graphy/4 }\end{array}$ & NA & $\begin{array}{l}\text { High performance } \\
\text { liquid chromatogra- } \\
\text { phy } \geq 4\end{array}$ & $74(19.22)$ & $82 \%$ & $53.60 \%$ & 7 & & $\begin{array}{r}\text { Eastern Me- } \\
\text { diterranean }\end{array}$ \\
\hline
\end{tabular}

PKU, phenylketonuria; WHO, World Health Organization; MS/MS, tandem mass spectrometry; ELISA, enzyme-linked immunosorbent assay; NA, not announced.

$1,10,11,24,27,28,33,34,44,46-48,50,53-60,62,63)$; of them, it was above $90 \%$ in 7 studies. ${ }^{11,27,34,46,47,57,59)}$ The participation rate increased with the progression of the screening process in 8 studies. $1,24,27,36,56$, $58,60,62)$

Moreover, 6 studies reported the percentage of consanguineous marriages among the parents of newborns with PKU. 1,13,16,17,21,50) The percentage of consanguineous marriages varied from $45 \%$ in Turkey $^{16)}$ to $100 \%$ in Iraq. ${ }^{13)}$

\section{Test characteristics}

In the included studies, 2 stages were used to diagnose infants with classical PKU.

\section{Screening tests}

A total of 19 studies reported the number of positive cases in the first stage of screening. The phenylalanine cutoff point for separating positive cases and referrals for diagnostic testing ranged from $1.65 \mathrm{mg} / \mathrm{dL}$ to $20 \mathrm{mg} / \mathrm{dL}$. The highest recall rate in the first stage of screening was 378 per 100,000 neonates in a study conducted in Russia. ${ }^{35)}$

\section{Diagnostic tests}

In the diagnostic stage, the phenylalanine cutoff point for diag. nosing classic PKU patients ranged from $1.65 \mathrm{mg} / \mathrm{dL}$ to $20 \mathrm{mg} /$ dL. Moreover, 22 studies selected $20 \mathrm{mg} / \mathrm{dL}$ as the positive cutoff point and 5 studies did not report a cutoff point. 2,9,16,22,25,26,31$36,43,44,46,51,55,57,58,62-64)$

\section{Pooled global prevalence of classic PKU}

Among the included studies, the highest prevalence was found in Turkey (38.13), followed by Iran, with a prevalence of 21.28 per 100,000 neonates, ${ }^{17,31)}$ while the lowest prevalence was reported in studies conducted in Thailand (0.3) and Taiwan $(0.44) .{ }^{42,4647,64)}$ 
A subgroup estimation of the polled prevalence showed that the pooled prevalence of classic PKU in the included studies was 6.002 (95\% CI, 5.07-6.93). The highest prevalence was seen in Eastern Mediterranean (9.83; 95\% CI, 6.18-13.48), Europe (8.11; 95\% CI, 6.54-9.69), Pan America (5.32; 95\% CI, 4.476.07), Western Pacific (2.94; 95\% CI, 0.91-4.97), and Southeast Asia (0.32; 95\% CI, 0.19-0.45) per 100,000 neonates (Fig. 2).

\section{Statistical analysis}

According to the results of mixed model, cutoff point selection had no effect on prevalence. The $p$ value obtained from the likelihood ratio test in the mixed model test suggested that the random intercept model was appropriate. Moreover, based on the intraclass coefficient, $29 \%$ of the PKU prevalence changes in different countries were justified by consideration of the WHO regions (Table 2).
A meta-regression test was used to assess the effect of year, phenylalanine cutoff point, region, neonate age at screening, and screening level (national or regional) on heterogeneity.

In the naïve model without any variables, $I^{2}$ was $99 \%$. Several models with different variables were created in which $I^{2}$ ranged was $97 \%-99 \%$, and the input of different variables did not decrease the heterogeneity.

Among the variables included in the model, only some WHO regions were significant. In the meta-regression model, the European region was selected as a reference. In the Eastern Mediterranean region, the prevalence was 1.01 greater than that in the European region, but the difference was not significant. The pooled prevalence of the different regions is reported in Table 3.

According to $I^{2}$ by region and overall, the studies had high heterogeneity (Table 4).

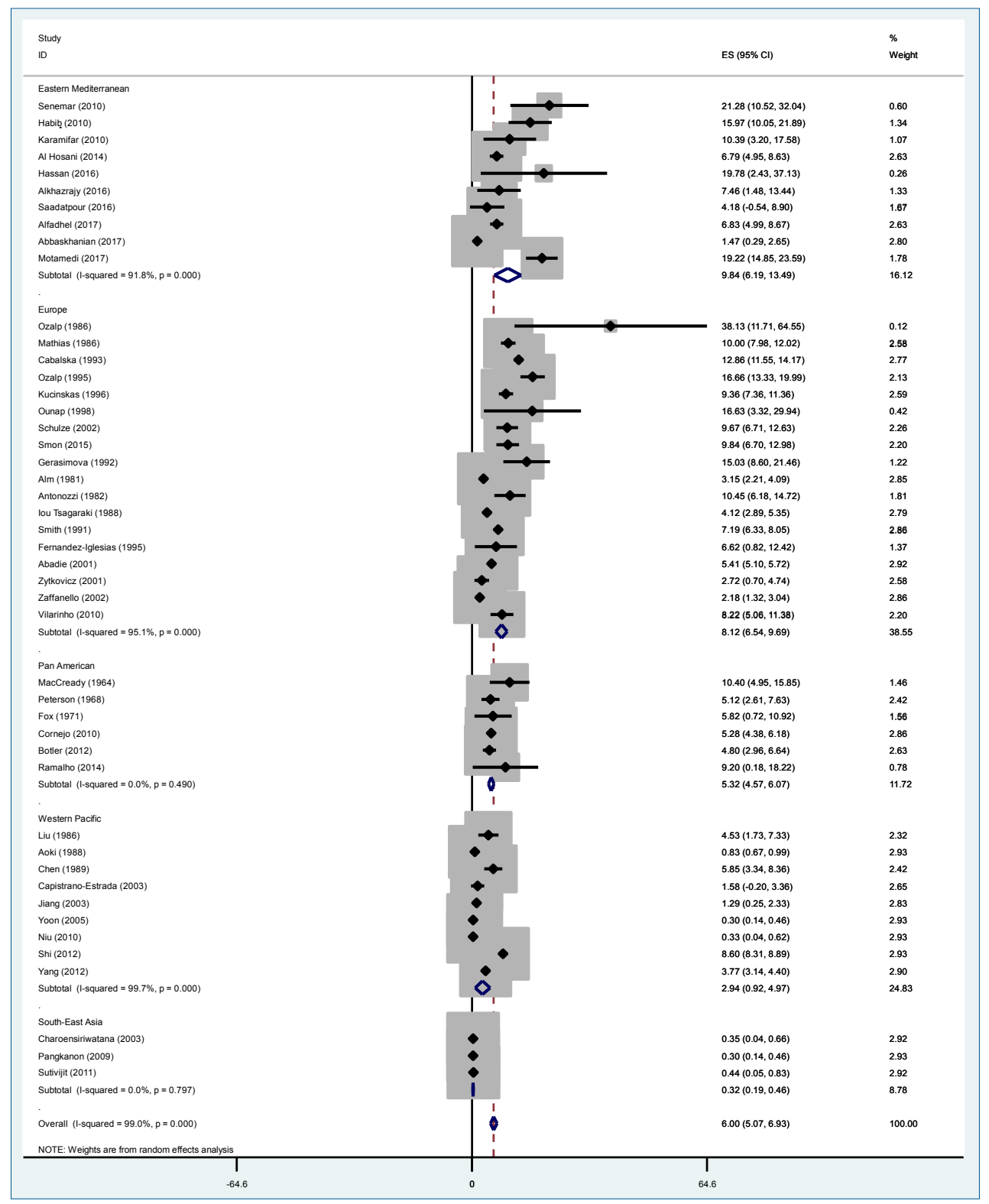

Fig. 2. Forest plot of pooled global prevalence of phenylketonuria. ES, estimated; $\mathrm{Cl}$, confidence interval. 
Table 2. Result of mixed model test

\begin{tabular}{|c|c|c|c|c|c|}
\hline Variable & Coefficient & SE & $P$ value & Estimate & $95 \% \mathrm{Cl}$ \\
\hline Cut-point level & -0.03 & 0.11 & 0.792 & & \\
\hline \multicolumn{6}{|l|}{ Random-effect parameter } \\
\hline WHO regions (var constant) & & 12.07 & & 15.61 & $3.43-71.08$ \\
\hline Var (residual) & & 8.09 & & 36.27 & $23.43-56.16$ \\
\hline
\end{tabular}

$\mathrm{SE}$, standard error; $\mathrm{Cl}$, confidence interval; WHO, World Health Organization.

Intraclass correlation coefficient $=0.29$. Likelihood-ratio test $=0.0019$.

Table 3. Result of meta-regression test

\begin{tabular}{|c|c|c|c|c|}
\hline WHO region & Coefficient & SE & $P$ value & $P^{2}$ \\
\hline Europe & Reference & & & \\
\hline Eastern Mediterranean & 1.01 & 1.89 & 0.593 & \\
\hline Pan American & -2.03 & 2.15 & 0.350 & $98.69 \%$ \\
\hline Western Pacific & -5.37 & 1.72 & 0.003 & \\
\hline Southeast Asia & -7.97 & 2.54 & 0.003 & \\
\hline
\end{tabular}

WHO, World Health Organization; SE, standard error.

Table 4. Prevalence rate and heterogeneity in regions

\begin{tabular}{|c|c|c|c|c|}
\hline WHO region & $P$ value & $P^{2}$ & $\begin{array}{l}\text { Prevalence in } 100000 \\
\text { neonates (range) }\end{array}$ & $\begin{array}{c}\text { Pooled prevalence in } \\
100,000 \text { neonates }\end{array}$ \\
\hline Pan American & 0.49 & 0 & $4.8-10.4$ & 5.32 \\
\hline Europe & $<0.0001$ & $95.1 \%$ & $2.18-38.13$ & 8.12 \\
\hline Western Pacific & $<0.0001$ & $99.7 \%$ & $0.3-8.6$ & 2.94 \\
\hline Southeast Asia & 0.79 & 0 & $0.3-0.44$ & 0.32 \\
\hline Eastern Mediterranean & $<0.0001$ & $91.8 \%$ & $1.47-21.28$ & 9.84 \\
\hline Overall & $<0.0001$ & $99 \%$ & $1.47-38.13$ & 6.002 \\
\hline
\end{tabular}

WHO, World Health Organization.

\section{Discussion}

This systematic review aimed to investigate the worldwide prevalence of PKU and provided a general picture of its status. The results of this study demonstrate that the worldwide prevalence of the disease is $0.3-38.13$ per 100,000 newborns. However, the meta-analysis revealed that the $\mathrm{I} 2$ index, which indicates heterogeneity, was reported for all regions except Southeast Asia (91.8\%) and Pan America (99.7\%), indicating high heterogeneity among countries and regions.

The uni- and multivariate models in the meta-regression showed that phenylalanine level, geographical area, neonate age at screening, screening level (national or regional), after the control for year of study, did not change heterogeneity.

However, the differences in prevalence can be attributed to 2 factors: (1) variability of the factors affecting disease worldwide; and (2) differences in the methods used in the studies.

PKU is a c0ngenital genetic disease; thus, factors such as culture, customs, consanguineous marriage, and genetics are expected to affect its incidence but among included studies only 6 studies in Iran, Iraq, Turkey, and the United Arab Emirates 1,13, 16,17,21,50) reported consanguineous marriages among parents of children with PKU.

Therefore, a lack of information about the prevalence of PKU in many countries in which consanguineous marriage is prevalent and a lack of reporting consanguineous marriage status in parents of children with PKU in many studies prevented us from controlling the effect of this variable on prevalence.

The next important determinant of prevalence is study performance; factors such as diagnostic tests, cutoff point, and sample size can affect the pooled prevalence in prevalence studies.

However, in the mixed model test, there was no significant relationship between cutoff point and disease prevalence, which might have been due to the effect of the confounding variables. However, the difference was noticeable when the cutoff point differed in the same population and within the same country. For example, in 3 studies conducted during 2000-2008 in Fars province (Iran), a different cutoff point was found. Moreover, Senemar chose a phenylalanine level of $\leq 4 \mathrm{mg} / \mathrm{dL}$ to define classical PKU and reported a prevalence of 21.28. ${ }^{17)}$ Habib et al. ${ }^{45)}$ considered a phenylalanine $\leq 10 \mathrm{mg} / \mathrm{dL}$ cutoff value and reported a prevalence of 15.97 . Furthermore, in the study of Karamifar et al., ${ }^{9)}$ phenylalanine levels above $20 \mathrm{mg} / \mathrm{dL}$ were considered positive and a prevalence of 10.39 was reported.

Sample size is the other factor involved in the difference in prevalence among studies. In a meta-analysis, the pooled prevalence is estimated according to the sample size, and larger studies have greater impact on prevalence. Thus, although studies conducted in the Eastern Mediterranean region reported higher prevalence than those in the Western Pacific region, 
since most studies in the latter had a larger sample size and the weighted sample size in that region was $24.83 \%$, higher than that in the Eastern Mediterranean region (16.2\%), the pooled prevalence in studies conducted in the Eastern Mediterranean region was close to that of the Western Pacific region.

Although this study addressed an important concern in genetic diseases, its findings may not be highly accurate, as there were many sources of heterogeneity in the reviewed studies that could have affected their pooled prevalence. Moreover, some heterogeneous sources might not have been identified. However, the standardization of study methods can partly solve this problem.

One of the limitations of this study was the failure to report consanguineous marriage in parents of newborns with PKU. Thus, it was not possible to answer the following question:

Is the difference in PKU prevalence among different countries due to differences in the number of consanguineous marriages?

Thus, we suggest that consanguineous marriages be recorded and reported in screening programs designed to identify patients with PKU and other congenital metabolic diseases.

In conclusion, all relevant studies conducted in 1964-2017 were included in this review. The highest PKU prevalence was observed in Turkey (38.13), while the lowest was seen in Thailand (0.3). Among the WHO regions, the highest prevalence belonged to Eastern Mediterranean Regional Office, while the lowest was in Southeast Asia. This difference in the prevalence may be due to differences in the number of consanguineous marriages among the different regions, phenylalanine cutoff points, and sample sizes.

\section{Conflicts of interest}

No potential conflicts of interest relevant to this article are reported.

\section{Acknowledgments}

The authors thank Moslem Taheri for his advice for extraction, Razieh Zahedi for her advice for the meta-analysis, Maryam Nazemzadeh for professional English editing.

\section{References}

1. Motamedi N, Goodarzi E, Pordanjani SR, Valizadeh R, Moradi Y, Sohrabivafa $\mathrm{M}$, et al. Incidence of phenylketonuria in Lorestan province, West of Iran (2006-2016). Int J Pediatr 2017;5:4713-21.

2. Šmon A, Grošelj U, Žerjav Tanšek M, Biček A, Oblak A, Zupančič M, et al. Newborn Screening in Slovenia. Zdr Varst 2015;54:86-90.

3. Souza CAA, Alves MRA, Soares RDL, Kanufre VC, Rodrigues VM, Norton RC, et al. $\mathrm{BH}(4)$ deficiency identified in a neonatal screening program for hyperphenylalaninemia. J Pediatr (Rio J) 2018;94:170-6.

4. Morovatdar N, Aval SB, Yazdi SMRH, Norouzi F, Mina T. The epidemiological and clinical study of Phenylketonuria (PKU) patients in
Khorasan, North-eastern Iran. Iran J Neonatol 2015;6:18-22.

5. Blau N, van Spronsen FJ, Levy HL. Phenylketonuria. Lancet 2010;376: 1417-27.

6. Mahmoud IG, Moneem MA, Mehany DA. Epidemiological study of neurometabolic diseases diagnosed at Cairo University Children Hospital: a two years outcome. Eur J Paediatr Neurol 2015;19(Suppl1):S121.

7. Liu J, Hoppman N, O'Connell JR, Wang H, Streeten EA, McLenithan JC, et al. A functional haplotype in EIF2AK3, an ER stress sensor, is associated with lower bone mineral density. J Bone Miner Res 2012;27:331-41.

8. Kitagawa T. Neonatal mass-screening for inborn errors of metabolism in Japan. Pediatr Int 1982;24:83-90.

9. Karamifar H, Ordoei M, Karamizadeh Z, Amirhakimi GH. Incidence of neonatal hyperphenylalaninemia in fars province, South iran. Iran J Pediatr 2010;20:216-20.

10. Botler J, Camacho LA, Cruz MM. Phenylketonuria, congenital hypothyroidism and haemoglobinopathies: public health issues for a Brazilian newborn screening program. Cad Saude Publica 2012;28:1623-31.

11. Dluholucký S, Knapková M. Newborn screening in Slovakia - from 1985 till today. Acta Facultatis Pharmaceuticae Universitatis Comenianae 2013;60:32-6.

12. Dluholucký S, Knapková M, Záhorcová M. First results from expanded newborn screening in Slovak Republic. Acta Facultatis Pharmaceuticae 2014;61:1-4.

13. Hamawandi AMH, Rashid JA, Raof Saeed HHMH, Hawrami OM. Annual incidence of phenylketonuria in Sulaimani City. Merit Res J Med Med Sci 2015;3:427-31.

14. Selim LA, Hassan SA, Salem F, Orabi A, Hassan FA, El-Mougy F, et al. Selective screening for inborn errors of metabolism by tandem mass spectrometry in Egyptian children: a 5 year report. Clin Biochem 2014; 47:823-8.

15. Tada K, Tateda H, Arashima S, Sakai K, Kitagawa T, Aoki K, et al. Followup study of a nation-wide neonatal metabolic screening program in Japan. A collaborative study group of neonatal screening for inborn errors of metabolism in Japan. Eur J Pediatr 1984;142:204-7.

16. Özalp I, Coşkun T, Tokatli A, Tokol S, Özgüç M, Köksal G, et al. Neonatal PKU screening in Turkey: 7 years experience in a developing country. Screening 1995;4:139-47.

17. Senemar S, Ganjekarimi H, Fathzadeh M, Senemar S, Tarami B, Bazrgar M. Epidemiological and clinical study of phenylketonuria (PKU) disease in the national screening program of neonates, Fars Province, Southern Iran. Iran J Public Health 2009;38:58-64.

18. Munn Z, Moola S, Lisy K, Riitano D. Joanna Briggs Institute reviewer's manual. Adelaide: The Joanna Briggs Institute, 2017.

19. Moher D, Liberati A, Tetzlaff J, Altman DG; PRISMA Group. Preferred reporting items for systematic reviews and meta-analyses: the PRISMA statement. Ann Intern Med 2009;151:264-9.

20. Hoy D, Brooks P, Woolf A, Blyth F, March L, Bain C, et al. Assessing risk of bias in prevalence studies: modification of an existing tool and evidence of interrater agreement. J Clin Epidemiol 2012;65:934-9.

21. Al-Jasmi FA, Al-Shamsi A, Hertecant JL, Al-Hamad SM, Souid AK. Inborn errors of metabolism in the United Arab Emirates: disorders detected by newborn screening (2011-2014). JIMD Rep 2016;28:12735 .

22. Farhud DD, Kabiri M. Incidence of phenylketonuria (PKU) in Iran. Indian J Pediatr 1982;49:685-8.

23. Hitzeroth HW, Niehaus CE, Brill DC. Phenylketonuria in South Africa. A report on the status quo. S Afr Med J 1995;85:33-6.

24. Zhong $\mathrm{K}$, Wang W, He F, Wang $\mathrm{Z}$. The status of neonatal screening in China, 2013. J Med Screen 2016;23:59-61.

25. MaCcready RA, Hussey MG. Newborn phenylketonuria detection program in Massachusetts. Am J Public Health Nations Health 1964;54: 2075-81.

26. Peterson RM, Koch R, Schaeffler GE, Wohlers A, Acosta PB, Boyle D. Phenylketonuria. Experience at one center in the first year of screening in California. Calif Med 1968;108:350-4.

27. Fox JG, Hall DL, Haworth JC, Maniar A, Sekla L. Newborn screening for hereditary metabolic disorders in Manitoba, 1965-1970. Can Med Assoc 
J 1971;104:1085-8.

28. Antonozzi I, Santagata G, Tofani R. Multiple neonatal screening for aminoacidopathies by ion exchange chromatography. Ric Clin Lab 1982; 12:507-15.

29. Liu SR, Zuo QH. Newborn screening for phenylketonuria in eleven districts. Chin Med J (Engl) 1986;99:113-8.

30. Mathias D, Bickel H. Follow-up study of 16 years neonatal screening for inborn errors of metabolism in West Germany. Eur J Pediatr 1986; 145:310-2.

31. Özalp I, Coşkun T, Ceyhan M, Tokol S, Oran O, Erdem G, et al. Incidence of phenylketonuria and hyperphenylalaninaemia in a sample of the Turkish newborn population. J Inherit Metab Dis 1986;9(Suppl 2):2379.

32. Missiou-Tsagaraki S, Soulpi K, Loumakou M. Phenylketonuria in Greece: 12 years' experience. J Ment Defic Res 1988;32(Pt4):271-87.

33. Chen RG, Pan XS, Qian DL, Guo H. Twenty-one cases of phenylketonuria out of 358,767 newborns in Shanghai, China. J Inherit Metab Dis 1989;12:485.

34. Smith I, Cook B, Beasley M. Review of neonatal screening programme for phenylketonuria. BMJ 1991;303:333-5.

35. Gerasimova NS, Samutin AA, Steklova IV, Tuuminen T. Phenylketonuria screening in Moscow using a microplate fluorometric method. Screening 1992;1:27-35.

36. Cabalska B, Nowaczewska I, Duczynska N, Laskowska-Klita T. Twentyfive years experience with newborn screening for phenylketonuria (PKU) in Poland. Screening 1993;2:29-32.

37. Fernández-Iglesias C, Flórez IG, Rodríguez-González MC, Gascón S. Neonatal screening for phenylketonuria and congenital hypothyroidism in Principado de Asturias (Spain) using two types of blood samples. Screening 1995;4:131-8.

38. Kucinskas V, Jurgelevicius V, Cimbalistiene L, Jusciene D, Smirnova M, Zamkauskiene D. Management and results of mass neonatal screening in Lithuania. Acta Medica Lithuanica 1996;3:38-42.

39. Zytkovicz TH, Fitzgerald EF, Marsden D, Larson CA, Shih VE, Johnson $\mathrm{DM}$, et al. Tandem mass spectrometric analysis for amino, organic, and fatty acid disorders in newborn dried blood spots: a two-year summary from the New England Newborn Screening Program. Clin Chem 2001; 47:1945-55.

40. Schulze A, Mayatepek E, Hoffmann GF. Evaluation of 6-year application of the enzymatic colorimetric phenylalanine assay in the setting of neonatal screening for phenylketonuria. Clin Chim Acta 2002;317:27-37.

41. Capistrano-Estrada S, Padilla CD. Hyperphenylalaninemia in the Philippines. Southeast Asian J Trop Med Public Health 2003;34 Suppl 3:1824.

42. Charoensiriwatana W, Janejai N, Boonwanich W, Krasao P, Chaisomchit S, Waiyasilp S. Neonatal screening program in Thailand. Southeast Asian J Trop Med Public Health 2003;34 Suppl 3:94-100.

43. Jiang J, Ma X, Huang X, Pei X, Liu H, Tan Z, et al. A survey for the incidence of phenylketonuria in Guangdong, China. Southeast Asian J Trop Med Public Health 2003;34 Suppl 3:185.

44. Yoon HR, Lee KR, Kang S, Lee DH, Yoo HW, Min WK, et al. Screening of newborns and high-risk group of children for inborn metabolic disorders using tandem mass spectrometry in South Korea: a three-year report. Clin Chim Acta 2005;354:167-80.

45. Habib A, Fallahzadeh MH, Kazeroni HR, Ganjkarimi AH. Incidence of phenylketonuria in Southern Iran. Iran J Med Sci 2010;35:137-9.

46. Niu DM, Chien YH, Chiang CC, Ho HC, Hwu WL, Kao SM, et al. Nationwide survey of extended newborn screening by tandem mass spectrometry in Taiwan. J Inherit Metab Dis 2010;33(Suppl 2):S295-305.

47. Sutivijit Y, Banpavichit A, Wiwanitkit V. Prevalence of neonatal hypothyroidism and phenylketonuria in Southern Thailand: A 10-year report. Indian J Endocrinol Metab 2011;15:115-7.

48. Alkhazrajy LA, Hassan AA. Overview of neonatal screening program applied at primary health care centers in Baghdad/Iraq. Int J Community
Coop Stud 2016;4:46-64.

49. Hassan FA, El-Mougy F, Sharaf SA, Mandour I, Morgan MF, Selim LA, et al. Inborn errors of metabolism detectable by tandem mass spectrometry in Egypt: The first newborn screening pilot study. J Med Screen 2016; 23:124-9.

50. Saadatpour Y, Dehghan F, Rasekhi S, Zolghadri N. Incidence of neonatal phenylketonuria in hormozgan province, Southern Iran, 2014-2016. J Global Pharm Technol 2016;12:509-14.

51. Abbaskhanian A, Zamanfar D, Afshar P, Asadpoor E, Rouhanizadeh H, Jafarnia A, et al. Incidence of neonatal hyperphenylalaninemia based on high-performance liquid chromatography confirmatory technique in Mazandaran Province, Northern Iran (2007-2015). Int J Prev Med 2017;8:93.

52. Alfadhel M, Al Othaim A, Al Saif S, Al Mutairi F, Alsayed M, Rahbeeni Z, et al. Expanded Newborn Screening Program in Saudi Arabia: Incidence of screened disorders. J Paediatr Child Health 2017;53:585-91.

53. Ounap K, Lilleväli H, Metspalu A, Lipping-Sitska M. Development of the phenylketonuria screening programme in Estonia. J Med Screen 1998;5:22-3.

54. Alm J, Larsson A. Evaluation of a nation-wide neonatal metabolic screening programme in Sweden 1965-1979. Acta Paediatr Scand 1981; 70:601-7.

55. Aoki K, Wada Y. Outcome of the patients detected by newborn screening in Japan. Acta Paediatr Jpn 1988;30:429-34.

56. Abadie V, Berthelot J, Feillet F, Maurin N, Mercier A, de Baulny HO, et al. Neonatal screening and long-term follow-up of phenylketonuria: the French database. Early Hum Dev 2001;65:149-58.

57. Zaffanello M, Zamboni G, Tatò L. Neonatal screening program for inborn errors of metabolism: a retrospective study from 1978 to 1997 in Northeastern Italy. Ita J Pediatr 2002;28:479-83.

58. Cornejo V, Raimann E, Cabello JF, Valiente A, Becerra C, Opazo M, et al. Past, present and future of newborn screening in Chile. J Inherit Metab Dis 2010;33 Suppl 3:S301-6.

59. Vilarinho L, Rocha H, Sousa C, Marcão A, Fonseca H, Bogas M, et al. Four years of expanded newborn screening in Portugal with tandem mass spectrometry. J Inherit Metab Dis 2010;33 Suppl 3:S133-8.

60. Shi XT, Cai J, Wang YY, Tu WJ, Wang WP, Gong LM, et al. Newborn screening for inborn errors of metabolism in mainland china: 30 years of experience. JIMD Rep 2012;6:79-83.

61. Yang LL, Mao HQ, Zhang WF, Zhao ZY, Yang RL, Zhou XL, et al. Pitfalls in the management of phenylketonuria in China. HK J Paediatr 2012;17:143-7.

62. Al Hosani H, Salah M, Osman HM, Farag HM, El-Assiouty L, Saade D, et al. Expanding the comprehensive national neonatal screening programme in the United Arab Emirates from 1995 to 2011. East Mediterr Health J 2014;20:17-23.

63. Ramalho AR, Ramalho RJ, Oliveira CR, Magalhães MM, Santos EG, Sarmento PM, et al. Evaluation of effectiveness and outcome of PKU screening and management in the State of Sergipe, Brazil. Arq Bras Endocrinol Metabol 2014;58:62-7.

64. Pangkanon S, Charoensiriwatana W, Janejai N, Boonwanich W, Chaisomchit S. Detection of phenylketonuria by the newborn screening program in Thailand. Southeast Asian J Trop Med Public Health 2009;40:525-9.

65. Schuler A, Somogyi C, Toros I, Nagy A, Kiss E, Varadi I, et al. Twenty years of experience with phenylketonuria in Hungary. Int Pediatr 1996;11: 114-7.

66. Ounap K, Lilleväli H, Klaassen T, Metspalu A, Sitska M. The incidence and characterization of phenylketonuric patients in Estonia. J Inherit Metab Dis 1996;19:381-2.

67. Pangkanon S, Ratrisawadi V, Charoensiriwatana W, Techasena W, Boonpuan K, Srisomsap C, et al. Phenylketonuria detected by the neonatal screening program in Thailand. Southeast Asian J Trop Med Public Health 2003;34 Suppl 3:179-81. 